\title{
Líquen plano esofágico
}

\author{
Esophageal lichen planus
}

\author{
Liquen plano esofágico
}

Janine Pichler de Oliveira, Luna Azulay-Abulafia*

\section{Resumo}

O líquen plano (LP) é uma doença inflamatória crônica autoimune mediada por linfócitos T que afeta pele, mucosas, unhas e couro cabeludo. O líquen plano esofágico (LPE) é uma manifestação de LP raramente relatada, apresentando-se comumente em mulheres de meia-idade e provocando sintomas como disfagia. Relatamos o primeiro caso de LPE no Brasil em mulher de 54 anos associado a líquen plano oral, cutâneo e ungueal. Embora o LP seja uma desordem bem conhecida pelos dermatologistas, relatos de LPE são raros na literatura dermatológica, sendo o LPE desconhecido pela maioria dos dermatologistas. Isto implica em um atraso significativo entre o início dos sintomas e o diagnóstico da doença.

Descritores: Esofagoscopia; Líquen plano; Endoscopia do sistema digestório.

\begin{abstract}
Lichen planus (LP) is a chronic immune-inflammatory disease that affects the skin, mucous membranes, nails, and scalp, mediated by T lymphocites. Esophageal lichen planus (LPE) is a rarely reported manifestation of LP, presenting commonly in middle-aged women and leading to symptoms such as dysphagia. Although LP is a disorder well known by dermatologists, reports of LPE are rare in dermatologic literature. The LPE is unknown by most dermatologists, with a significant delay in the diagnosis of the disease.
\end{abstract}

Keywords: Esophagoscopy; Lichen planus; Endoscopy, digestive system.

\section{Resumen}

El liquen plano (LP) es una enfermedad inflamatoria crónica autoinmune mediada por linfocitos T que afecta piel, mucosas, uñas y cuero cabelludo. El liquen plano esofágico (LPE) es una manifestación de LP raramente mencionada, se presenta comúnmente en mujeres de media edad, a través de sintomas como disfagia. Describimos el primer caso de LPE en el Brasil, en una mujer de 54 años relacionada con el liquen plano oral, cutáneo y ungueal. Aunque LP sea un desorden muy conocido por los dermatologistas, las citaciones sobre el LPE son raras en la literatura dermatológica. El LPE es desconocido por la mayoría de los dermatologistas. Esto implica un atraso significativo entre el inicio de los sintomas y el diagnóstico de la enfermedad.

Palabras clave: Esofagoscopía; Liquen plano; Endoscopía del sistema digestivo. 


\section{Introdução}

O líquen plano (LP) é uma doença inflamatória crônica autoimune mediada por linfócitos $\mathrm{T}$ que acomete pele, mucosas, unhas e couro cabeludo. Afeta 0,5 a $2 \%$ da população e tem maior prevalência em mulheres entre a quarta e quinta década de vida. ${ }^{1,2,3,4}$ O líquen plano esofágico (LPE) é uma manifestação de LP raramente relatada e que comumente se apresenta em mulheres de meia-idade, como disfagia secundária a lesões esofágicas superiores e estenoses, na presença de líquen plano oral (LPO). ${ }^{5,6,7}$ O LPE é uma entidade reconhecida recentemente. ${ }^{8,9}$ Em 1982, Guedon e colaboradores ${ }^{9}$ relataram o caso de um paciente com estenose do terço médio do esôfago, inicialmente pensando ser secundário ao refluxo gastroesofágico, mas o diagnóstico foi corrigido para LPE após o desenvolvimento do LPO erosivo. Em 1982, Al-Shihabi-Jackson ${ }^{7}$ e Lefer, ${ }^{10}$ simultaneamente, mas de forma independente, relataram os primeiros casos de LPE na literatura inglesa. Em 1983, Ottingnon e colaboradores ${ }^{11}$ relataram um paciente com disfagia causada por uma estenose do terço inferior do esôfago e que posteriormente desenvolveu LPO. ${ }^{8}$

Embora o LP seja uma desordem bem conhecida pelos dermatologistas, relatos de LPE são raros na literatura dermatológica. O objetivo desse artigo é informar e alertar sobre a existência do LPE na presença do LPO para que o diagnóstico do acometimento esofágico seja feito e, assim, sejam minimizados os danos decorrentes do curso prolongado desta doença.

\section{Estratégia de busca}

Foram revisados no PubMed relatos de LPE até abril de 2013. A busca foi feita pelas palavras-chave "esophageal lichen planus" e "esophagus lichen planus". Bibliografias dos relatos encontrados foram revisadas, e suas referências cruzadas. Relatos de casos foram avaliados pela idade de início, sexo, localização do LP, relação entre o início do LPE e do LP extraesofágico, achados endoscópicos, biópsia e histopatologia, história médica (incluindo história gastrointestinal), desenvolvimento de carcinoma espinocelular (CEC) no esôfago, terapias e resposta ao tratamento.
Apresentamos uma revisão sistemática dos 82 casos descritos na literatura até abril de 2013.

A revisão revelou 82 casos de LPE: 72 na literatura inglesa, nove na literatura europeia (quatro franceses, ${ }^{9,12,13,14}$ um alemão, ${ }^{15}$ dois italianos ${ }^{16,17} \mathrm{e}$ dois espanhóis). ${ }^{18,19}$

Relatamos o primeiro caso de LPE descrito no Brasil, já aceito para publicação. ${ }^{20}$

As tabelas de 1 a 6 retratam as características dos 82 pacientes.

\section{Prevalência}

A prevalência do LPE é desconhecida e comumente subestimada. Nossa revisão encontrou 12 pacientes (15\%) com doença assintomática, cujo diagnóstico de LPE foi feito pela endoscopia.

Dickens e colaboradores ${ }^{21}$ avaliaram 19 pacientes por endoscopia que possuíam o diagnóstico de LP. Cinco dos 19 pacientes tinham LPE. $\mathrm{O}$ autor concluiu que, embora o estudo fosse pequeno, o achado de $25 \%$ de prevalência indicava que o LPE era amplamente subestimado. Menges e colaboradores ${ }^{22}$ sugeriram que embora o LPE sintomático ocorra em menos de $1 \%$ dos pacientes com LP, o envolvimento assintomático do esôfago tende a ser mais comum do que atualmente relatado, e a presença de sintomas de doença esofágica apresenta uma correlação íntima com a presença de LPE ao exame endoscópico.

Eisen $^{23}$ avaliou 584 pacientes com diagnóstico de LP oral e manifestações extraorais. Quatro de oito pacientes com disfagia tinham achados endoscópicos consistentes com LP que foram confirmados pela histopatologia. Todos os pacientes desenvolveram doença esofágica após o início das lesões orais. Este autor suspeitou que, por ter avaliado por endoscopia somente aqueles pacientes que relatavam disfagia, eles provavelmente perderam casos assintomáticos de doença esofagiana. Quispel e colaboradores ${ }^{24}$ prospectivamente rastrearam 24 pacientes com LP para envolvimento esofágico usando cromoendoscopia de alta resolução e biópsia esofágica de fragmentos proximal, médio e distal do esôfago e constataram que 50\% (12 de 24) dos pacientes tinham LPE.

Estes dados sugerem que o envolvimento esofágico com LP não é raro e pode ser assintomático. Médicos que cuidam de pacientes com 
LP devem estar conscientes das características clínicas do envolvimento esofágico, especialmente em pacientes com múltiplos sítios de LP.

\section{Apresentação clínica}

LP de mucosas tendem a ter maior prevalência em mulheres. No estudo de 723 pacientes com LP oral, 75\% eram mulheres, 25\% eram homens e a média de idade de aparecimento do LP oral em mulheres foi de 57 anos..$^{25} \mathrm{Em}$ nossa revisão, o LPE teve uma importante predominância em mulheres de meia-idade, com 89\% (64 de 73) dos pacientes, sendo mulheres com média de idade de 61,9 anos. Somente oito dos pacientes relatados com LPE eram homens (Tabela 1).

O LPE afeta preferencialmente pacientes com LP oral e/ou vulvar. O LP extraesofágico esteve presente em 99\% (81 de 82) dos pacientes, mais comumente LP oral (89\%) e vulvar (42\%) (Tabela 2).

Os sintomas mais comuns de LPE são disfagia (82\% [67/82]), odinofagia (32\% [26/82]) e perda de peso (23\% [19/82]). Outros sintomas que sugerem envolvimento esofágico incluem queimação retroesternal, rouquidão, asfixia e dor epigástrica (Tabela 3). Particularmente, é importante salientar que sintomas como dor epigástrica e refluxo mudando para odinofagia anunciam uma transformação maligna para CEC..$^{26,27}$

Sintomas esofágicos podem preceder, aparecer simultaneamente, ou surgir após o diagnóstico de LP cutâneo (Figura 1), oral (Figura 2) ou genital.

Doença oral, cutânea ou genital anteceden-

Tabela 1. Característica dos pacientes.

\begin{tabular}{l|r}
\hline Total do no de pacientes & 82 \\
\hline № de pacientes com gênero descrito & 73 \\
F & $64(89 \%)$ \\
M & $8(11 \%)$ \\
F:M & $8: 1$ \\
No de pacientes com idade relatada & $72(64 \mathrm{~F}, 8 \mathrm{M})$ \\
Idade do diagnóstico (anos) & $22-85$ \\
Média de idade dos pacientes F (anos) & 61,9 \\
Média de idade dos pacientes M (anos) & 29,5 \\
\hline
\end{tabular}

Legenda: $F$, feminino; $M$, masculino. do sintomas esofágicos foram relatadas em 33 pacientes (40\%) do estudo. Na revisão, notou-se que 14 pacientes desenvolveram LPE como única ou primeira manifestação de LP.

\section{Doenças associadas}

Nosso estudo não foi capaz de determinar a prevalência de outras doenças associadas com LPE porque a maioria dos pacientes não tinha uma história médica consistente mencionada. Os pacientes estudados nesta revisão tiveram doenças de tireoide como as mais comumente relatadas (Tabela 5). Embora hepatite infecciosa, especialmente hepatite $C$, tenha sido associada com LP, ${ }^{26,27}$ não há dados suficientes associando hepatite infecciosa e LPE. Em nossa revisão somente um paciente tinha hepatite $\mathrm{C}$.

\section{Achados endoscópicos}

Os endoscopistas dividem o esôfago em terços: o terço superior correspondente aos $20 \mathrm{~cm}$ a partir dos incisivos e que se encontra acima do arco aórtico, o terço médio, que se estende até cerca de $30 \mathrm{~cm}$; e o terço distal, chegando até os $40 \mathrm{~cm}$ a partir dos incisivos.

Na revisão, a localização das lesões no esôfago foi mencionada em $92 \%$ (75 de 81) dos pacientes, com algum tipo de envolvimento proximal ocorrendo em 90\% (68 de 75) dos pacientes (Tabela 6). Observar o local dos achados endoscópicos é, portanto, fundamental para fazer o diagnóstico do LPE, já que a doença geralmente afeta as porções proximal e média do esôfago, enquanto a esofagite de refluxo é sempre distal. Importante característica que auxilia na diferenciação do LPE da doença do refluxo gastroesofágico (DRGE) é a preservação da junção gastroesofágica no LPE, ${ }^{26}$ apesar de um caso de LPE em nossa análise ter se estendido ao esôfago distal logo acima da junção gastroesofágica.

A descrição dos resultados da endoscopia do esôfago variam e, às vezes, são descritos como "sutil", ${ }^{28}$ mas caracteristicamente incluem: descamação, como pseudomembranas; ${ }^{28}$ friabilidade e sangramento da mucosa inflamada, especialmente após a passagem do endoscópio; ${ }^{29}$ e pápulas e placas submucosas..$^{30}$ Outros achados sugestivos incluem placas rendilhadas brancas, erosões, ul- 
Tabela 2. Presença de líquen plano extraesofágico.

\begin{tabular}{l|r}
\hline Achados de líquen plano extraesofágico & \multicolumn{1}{|r}{$81 / 82(99 \%)$} \\
\hline Oral & $72 / 81(90 \%)$ \\
Vulvovaginal/anogenital & $39 / 81(49 \%)$ \\
Cutâneo & $36 / 81(45 \%)$ \\
Couro cabeludo & $5 / 81(6 \%)$ \\
Unha & $3 / 81(4 \%)$ \\
Ocular & $1 / 81(1 \%)$ \\
Localização não especificada & $1 / 81(1 \%)$ \\
\hline
\end{tabular}

Tabela 3. Sintomas associados ao líquen plano esofágico.

\begin{tabular}{l|r}
\hline Sintomas esofagianos relatados & $82 / 82(100 \%)$ \\
Disfagia & $67 / 82(82 \%)$ \\
Odinofagia & $26 / 82(32 \%)$ \\
Assintomático & $21 / 82(26 \%)$ \\
Perda de peso & $19 / 82(23 \%)$ \\
Queimação retroesternal & $6 / 82(7 \%)$ \\
Regurgitação & $2 / 82(2 \%)$ \\
Asfixia & $1 / 82(1 \%)$ \\
Rouquidão & $1 / 82(1 \%)$ \\
\hline
\end{tabular}

cerações e estenoses. Quispel e colaboradores ${ }^{30}$ relataram que em 12 pacientes com LPE o diagnóstico foi feito através de biópsia das mucosas de aparência normal.

Tramas, estenoses, estreitamentos e constrições foram algumas vezes documentadas durante o curso da doença em 53\% (43 de 82) dos casos revisados.

Estenoses esofágicas foram descritas como "anel esbranquiçado reticulado ou tramas aparentes" e "frequentemente múltiplas em diferentes níveis do esôfago". 28

A duração do desenvolvimento dos sintomas de estenose foi de cinco a seis anos em alguns casos. ${ }^{6,22}$ Se uma estenose não está presente na avaliação inicial, muitas vezes pode se desenvolver ao longo do curso da doença; ou seja, alguns casos demonstram a progressão das mudanças de pseudomembranas, erosões ou tramas para estenoses com aberturas estreitas, impedindo pacientes de se alimentarem e resultando em perda de peso não intencional.
Tabela 4. Relação entre o início do líquen plano extraesofágico e do LPE.

\begin{tabular}{l|r}
\hline $\begin{array}{l}\text { Início das lesões extraesofágicas antes } \\
\text { dos sintomas esofagianos }\end{array}$ & 33 pacientes \\
\hline $\begin{array}{l}\text { Líquen plano esofágico como única ou } \\
\text { primeira manifestação de LP }\end{array}$ & 14 pacientes \\
\hline
\end{tabular}

Tabela 5. Doenças relatadas em associação com LPE.

\begin{tabular}{l|c}
\hline Doença & № de pacientes* \\
\hline Doença da tireoide (qualquer tipo) & 8 \\
Nódulo benigno da tireoide & 1 \\
Bócio difuso & 1 \\
Hipertireoidismo & 1 \\
Hipotireoidismo & 1 \\
$\begin{array}{l}\text { Ressecção da glândula tireoide } \\
\text { (causa desconhecida) }\end{array}$ & 2 \\
$\begin{array}{l}\text { Substituição hormonal da tireoide } \\
\text { (causa desconhecida) } \\
\text { Hepatite C }\end{array}$ & 2 \\
\hline *Denominador referente ao número de pacientes testados.
\end{tabular}

O esôfago proximal é a localização majoritária das lesões do LPE, podendo ser de difícil avaliação, pois pacientes tendem a rejeitar o instrumento quando se está próximo à região do esfíncter esofágico superior. ${ }^{5}$ Além disso, o endoscopista suspeita o diagnóstico que é mais provável e comum: no caso, DRGE, podendo "esquecer" das lesões proximais do paciente com queixa de disfagia, concentrando-se no terço médio e distal do esôfago, onde lesões estruturais e distúrbios da motilidade são usualmente identificados. ${ }^{11,31,27}$ Do ponto de vista dos endoscopistas, recomenda-se que uma história dermatológica e um exame da orofaringe completos sejam sempre realizados, para que lesões esofágicas proximais sejam encontradas na endoscopia. ${ }^{31,27}$

\section{Histopatologia}

A histopatologia foi descrita em 98\% (80 de 82) dos pacientes revisados. Achados histopatológicos encontrados "consistentes com" LPE estiveram presentes em $74 \%$ dos casos (61 de 82).

A revisão da literatura revela que, ao contrário das lesões de LP cutâneo, a histopatologia do 
Tabela 6. Achados endoscópicos do LPE.

\begin{tabular}{l|c}
\hline Endoscopia realizada & $82 / 82(100 \%)$ \\
Localização das lesões mencionadas & $76 / 82(93 \%)$ \\
Esôfago médioproximal & $52 / 76(68 \%)$ \\
Esôfago distal & $17 / 76(22 \%)$ \\
Proximal e distal & $27 / 76(36 \%)$ \\
Histologia descrita & $80 / 82(98 \%)$ \\
“Consistente com” LPE & $61 / 82(74 \%)$ \\
Displasia ou CEC & $14 / 82(17 \%)$ \\
Estenose & $44 / 82(54 \%)$ \\
\hline
\end{tabular}

LPE é altamente variável e muitas vezes inconclusiva, ${ }^{12,32}$ com diagnósticos histopatológicos inespecíficos como "esofagite" ou "inflamação crônica" sendo comuns. No entanto, no contexto clínico correto e com características histopatológicas sugestivas, o médico é capaz de chegar a um diagnóstico definitivo.

Há pouca literatura disponível sobre a histopatologia do LPE. Em geral, as características histopatológicas de LPE mais se assemelham às conclusões de LP oral do que LP cutâneo. ${ }^{3}$ Quando o LP afeta membranas mucosas, o epitélio pode ser perdido. ${ }^{10,22} \mathrm{O}$ esôfago carece de uma camada granular e camada córnea. A hipergranulose é muitas vezes ausente e a hiperqueratose, se presente em toda a extensão do epitélio, será composta por paraqueratose em vez de ortoqueratose. ${ }^{3,10}$ Infiltrado denso em banda linfocítica na lâmina própria, degeneração da camada basal e corpos de Civatte (queratinócitos necróticos) estão tipicamente presentes., 3,12,19,32 Depósitos de lgM subepidérmico detectados em imunofluorescência direta são altamente característicos, mas não garantem diagnóstico de LP. 27,28

\section{Diagnóstico diferencial}

O diagnóstico diferencial do LPE pode ser dividido em duas categorias distintas: condições que envolvem primariamente o esôfago e desordens cutâneas que podem envolver o esôfago.

De longe, a doença esofágica primária mais importante a ser excluída é a DRGE, já que apresenta sintomas comuns ao LPE. ${ }^{3}$ A esofagite descamativa do LP, que afeta o esôfago inferior, pode ser incorretamente interpretada como secundária à esofagite de refluxo. ${ }^{27,28}$ Certas características endoscópicas podem ajudar a distinguir os dois acometimentos. A localização pode ajudar a excluir o refluxo, já que o LPE geralmente afeta o esôfago superior, enquanto o refluxo sempre afeta o terço inferior do esôfago. ${ }^{22}$ Curiosamente, Quispel e colaboradores ${ }^{24}$ observaram uma alta prevalência de esofagite ( 8 de 12) em seus pacientes com LPE e, portanto, sugerem que o próprio LP pode predispor a esofagite.

Estenoses não são achados específicos de LPE nem de esofagite de refluxo. Estenoses causadas por refluxo ocorrem geralmente na junção gastroesofágica associadas com esofagite erosiva e ulcerações lineares. Estas alterações não são observadas no LPE. Além disso, se a lesão é no terço superior do esôfago, com os achados macroscópicos e histopatológicos distais normais à zona de envolvimento, a esofagite de refluxo torna-se muito menos provável. ${ }^{22}$ Em casos difíceis, refluxos também podem ser excluídos por falta de sintomas clínicos, resultados negativos dos estudos de sonda de $\mathrm{pH}$ e falta de resposta à terapia antirrefluxo.

O diagnóstico diferencial de doenças que potencialmente envolvem tanto a pele quanto o esôfago é extenso e listado no quadro 1.

\section{Potencial de evolução para malignidade}

Considerar o LP como uma condição prémaligna é algo controverso e dados que o confirmem como um fator de risco independente para o desenvolvimento de CEC são escassos; 27,33 entretanto, o risco de transformação maligna de LP oral tem sido relatado com taxas que variam de 0,6 a $5,32 \%,{ }^{2,25,33-36}$ e seu desenvolvimento ocorre entre 3,4 a 6,5 anos após o início do LP. 33,35 Em comparação, nos Estados Unidos, o CEC é responsável por 85 a $90 \%$ dos 5.000 novos casos por ano de câncer na orofaríngea. ${ }^{37} \mathrm{O}$ CEC em desenvolvimento no cenário da LP parece diferir do CEC que ocorre na população em geral, em que $90 \%$ dos pacientes são homens com mais de 45 anos e fatores de risco concomitantes, como álcool e/ou tabaco. ${ }^{38}$

O papel do tabagismo e do álcool no desenvolvimento de CEC oral nos pacientes com LP 


\section{Quadro 1. Diagnóstico diferencial de LPE.}

\begin{tabular}{|l|}
\hline Somente esôfago \\
\hline EsGE \\
Infecções fúngicas (cândida e outras) \\
Infecções virais (HSV, EBV, CMV) \\
Doença de Chagas \\
\hline Esôfago e pele \\
\hline $\begin{array}{l}\text { Buloses: pênfigo vulgar, pênfigo paraneoplásico, epider- } \\
\text { mólise bolhosa adquirida, pênfigo de membrana mucosa, } \\
\text { pênfigoide bolhoso, doença de Hailey-Hailey } \\
\text { Reações medicamentosas: cianamida, tiazídico, anti- } \\
\text { malárico, sais de ouro }\end{array}$
\end{tabular}

Doenças autoimunes: doença de Behçet, esclerodermia, dermatomiosite

Neoplasia: síndrome de Bazex, melanoma metastático, tilose

Síndrome de Steven-Johnson

Necrólise epidérmica tóxica

Líquen escleroso

Telangiectasia hemorrágica hereditária

Doença enxerto $\mathrm{x}$ hospedeiro

Acantose nigricante

Disceratose congênita

Doença de Crohn

Legenda: CMV, Citomegalovírus; EBV, Epstein-Barr vírus; HSV, Herpes simples vírus.

não foi determinado. Estes fatores de risco foram notavelmente ausentes em alguns pacientes na literatura com LP oral associado a CEC. ${ }^{35} \mathrm{O}$ exato mecanismo da degeneração maligna do LP oral para CEC é desconhecido. Possíveis fatores contribuem, incluindo o aumento do turnover das células basais, diminuição da vigilância imune e/ ou a carcinogênese vírus-induzida promovida por imunossupressores usados no tratamento do LP oral. ${ }^{26}$

O CEC que surge no cenário do LP oral tem sido observado por ocorrer em lesões orais préexistentes e na mucosa oral afetada de pacientes com LP.,35-37 Além disso, um estudo sugere que o desenvolvimento de CEC na mucosa de pacientes com LP mucoso pode indicar um risco mais elevado de um segundo CEC, e que este aumento do risco refere-se a todas as mucosas afetadas por LP. ${ }^{38}$ Esta constatação é especialmente sobre dados dos relatos de CEC esofágico em pacientes com LPE.

$\mathrm{O}$ fato de que a transformação maligna do LPE foi avaliada em quatro doentes sugere que o LPE também possa ser uma condição prémaligna. ${ }^{26,27,33,37}$ A mucosa esofágica no LPE é frequentemente erosiva, uma característica associada com a transformação maligna no LP oral. $34,35,39$

O CEC é responsável por 30 a $40 \%$ dos casos de câncer de esôfago nos países desenvolvidos. ${ }^{39}$

Em 2003, Calabrese e colaboradores ${ }^{26}$ relataram o primeiro caso de transformação de uma lesão de LPE para CEC em uma mulher de 49 anos, nove anos e 11 meses depois de sua apresentação inicial e diagnóstico de LPE. Este é um caso particularmente importante por várias razões. Em primeiro lugar, foi o primeiro relato clínico e histopatologicamente documentado de uma lesão de LPE transformada em CEC. Em segundo lugar, a reavaliação, solicitada pelo novo sintoma de odinofagia da paciente, levou a resultados muito marcantes: a endoscopia mostrou uma massa de esôfago endofítica no local exato onde três meses antes havia uma lesão de espessamento da mucosa com histologia negativa; a tomografia computadorizada demonstrou uma subestenose, $6 \mathrm{~cm}$ de tumor no tórax, abdômen e pescoço, e toracotomia expôs uma massa, irressecável no terço proximal do esôfago com infiltração mediastinal.

O caso relatado por Calabrese e colaboradores $^{26}$ destaca de forma precisa que as lesões de LPE podem sofrer degeneração maligna com graves consequências, apesar de sintomas mínimos, enfatizando a necessidade de um elevado índice de suspeição e acompanhamento de perto em todos os pacientes com LPE, especialmente quando há uma alteração dos sintomas. Esta recomendação preliminar é ainda mais fundamentada pelos primeiros casos familiares de LPE e o desenvolvimento de câncer de esôfago em duas irmãs com 


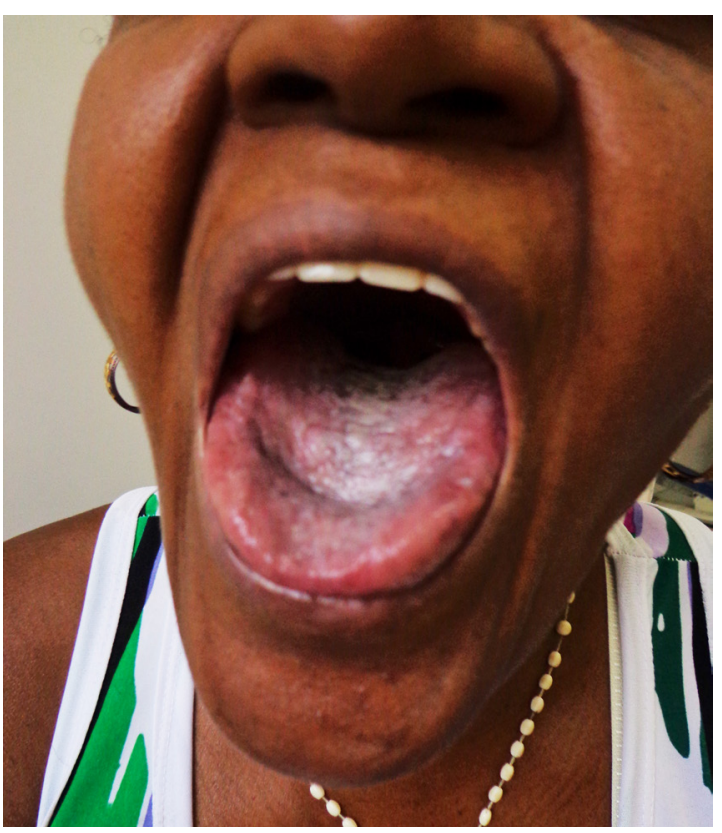

Figura 1. Estrias finas, brancas de aspecto rendilhado sobre o dorso da língua.

idades entre 68 e 70 anos. ${ }^{36}$ Ambas tiveram longo curso de LP cutâneo e mucosa, e disfagia. Uma irmã apresentou disfagia progressiva e foi dado o diagnóstico de CEC T3NO (T3: tumor invade a adventícia e N0: sem metástases para linfonodos regionais). Isso fez com que a outra irmã procurasse uma avaliação sem quaisquer alterações dos seus sintomas, resultando na detecção de um incidental achado de CEC T1NO (T1: tumor invade a lâmina própria ou submucosa e NO: sem metástases para linfonodos regionais). Estes autores fazem um adendo: talvez o CEC associado com LP tenha curso relativamente benigno, pois seus pacientes apresentaram disfagia prolongada e a histologia de ambos CECs não era invasiva. O caso relatado por Calabrese e colaboradores, ${ }^{26}$ no entanto, contradiz esta sugestão.

Vários autores sugerem que, embora a triagem endoscópica para displasia em todos os pacientes com LP não possa ser justificada, a endoscopia deve ser realizada em pacientes com LP que tenham sintomas sugestivos de envolvimento do esôfago e que a investigação possa levar à descoberta precoce da displasia. ${ }^{39}$ Apoiamos esta abordagem, uma vez que é provavelmente o método diagnóstico mais adequado e eficaz em termos de custos para detectar a doença precocemente, implementar terapia e evitar os resultados negativos (constrições, degeneração maligna).

\section{Tratamento}

A natureza rara do LPE faz com que estudos clínicos randomizados para avaliação da eficácia terapêutica sejam muito difíceis, de modo que os dados mais seguidos são os dos relatos e séries de casos. Muitos pacientes requerem um tratamento multidisciplinar, com terapias sistêmicas em conjunto com endoscopias regulares e dilatações quando necessárias, para sucesso terapêutico.

Terapias para LPE têm incluído corticoides sistêmicos, ciclosporina, azatioprina e retinoides sistêmicos (etretinato e acitretina). ${ }^{3,7}$ Em nossa revisão, esteroides sistêmicos foram os mais efetivos na dose de 40 a $60 \mathrm{mg} /$ dia por quatro a seis semanas. Uma resposta foi usualmente notada entre uma e duas semanas. Alguns pacientes tiveram recorrência dos sintomas quando a primeira dose foi reduzida. ${ }^{22}$ Ciclosporina na dose de 3 $\mathrm{mg} / \mathrm{kg} / \mathrm{dia}^{27}$ e rituximab ${ }^{29}$ têm sido documentados como efetivos.

Embora os pacientes possam sentir um alívio sintomático com corticosteroides ou outros imunossupressores, muitas vezes ainda requerem repetidas dilatações. ${ }^{31}$ Os resultados suportam a avaliação agressiva e a intervenção precoce para o LPE em pacientes sintomáticos, a fim de evitar cicatrizes a longo prazo e formação de estenoses.

Outras opções terapêuticas a serem consideradas podem incluir tacrolimus sistêmico, micofenolato mofetil, interferon $a-2 b$, agentes biológicos imunomoduladores. ${ }^{40}$

A presença de LPE concomitantemente em áreas com esofagite de refluxo pode ser um exemplo do fenômeno de Koebner. ${ }^{9} \mathrm{O}$ tratamento adjuvante da prevenção de refluxo visando a proteção do epitélio pode evitar ainda mais dano. ${ }^{22}$ Em alguns casos, a intolerância a medicação ou efeitos colaterais requerem uma estreita vigilância endoscópica com dilatações intermitentes no lugar da terapia. ${ }^{3}$

\section{Discussão}

O líquen plano é uma doença inflamatória crônica que afeta a pele, mucosas, unhas e couro cabeludo. ${ }^{1}$ Nossa paciente apresentava LP de pele, unhas e mucosa, com confirmação histo- 


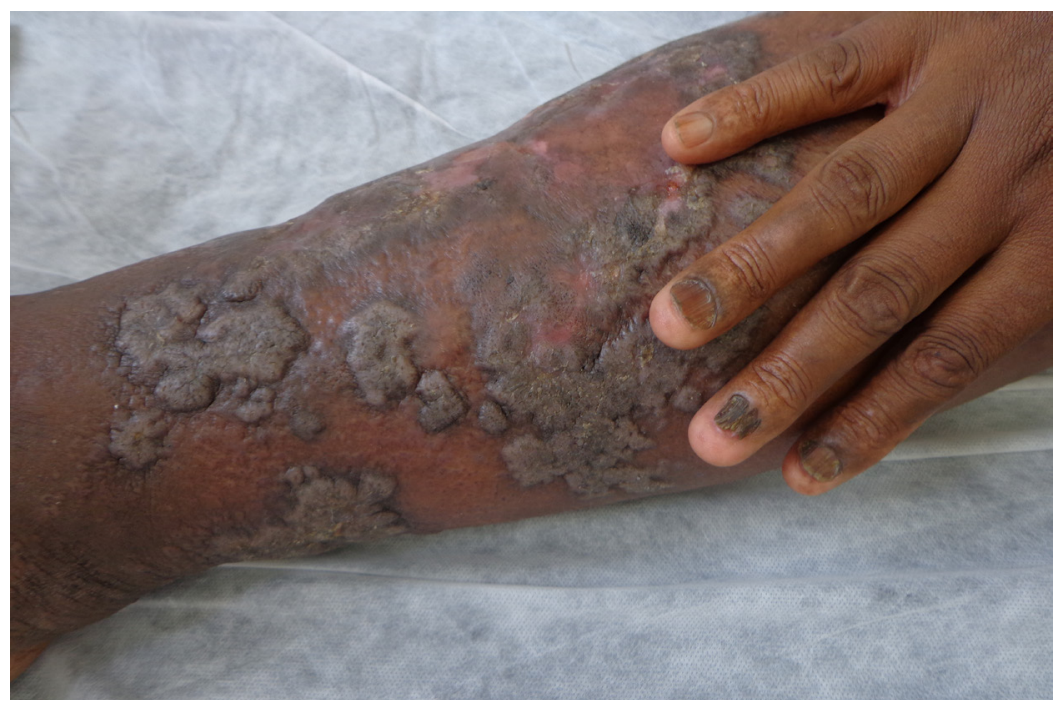

Figura 2. Na região pré-tibial esquerda, placas hipertróficas (líquen plano hipertrófico). Ainda lesões ungueais de líquen plano (mesma paciente da figura 1).

patológica.

A verdadeira prevalência do LPE na população geral é quase impossível de determinar, por várias razões, como: desconhecimento clínico e patológico da condição, alta taxa de doença assintomática ou sutil, e a possibilidade de que o envolvimento do esôfago seja a única manifestação de LP.3,6,7,8,9

Em revisão de 82 casos publicados na literatura sobre LPE, notou-se predominância marcante de mulheres de meia-idade entre os pacientes, sendo afetados especialmente aqueles com LP oral e/ou vulvar, apresentação compatível com os dados da nossa paciente. O LP extraesofágico pode estar presente em até 99\% dos pacientes, sendo mais comumente observados na cavidade oral e na vulva; em nosso relato a paciente apresentava LP oral, porém não vulvar. Os sintomas mais comuns são disfagia, odinofagia e perda de peso; no presente caso a paciente referia principalmente o sintoma de disfagia. ${ }^{3}$ Outros sintomas que sugerem o envolvimento do esôfago incluem rouquidão, engasgos e dor epigástrica. ${ }^{27}$

Apesar das doenças da tireoide serem as doenças mais comumente associadas ao LPE, no nosso caso não havia tireoidopatias.

Embora hepatites infecciosas, especialmente a hepatite $\mathrm{C}$, tenham sido relatadas em associação com LP, não existem dados especificamente associando hepatite infecciosa com LPE. ${ }^{3}$ Nossa paciente tinha sorologia negativa para hepatite $\mathrm{C}$.
Na endoscopia digestiva alta foram observadas estrias brancas de aspecto rendilhado no terço superior do esôfago como descritas na literatura. A biópsia do esôfago demonstrou córion com moderado infiltrado inflamatório linfocitário confluente no escasso estroma conjuntivo presente e entre os queratinócitos basais do epitélio escamoso, com alguns queratinócitos apoptóticos, semelhantes a casos relatados na literatura.

Como LPE é uma condição potencialmente pré-maligna para o carcinoma de células escamosas, o paciente deverá ser submetido à endoscopias a cada um ou dois anos, principalmente aqueles com LP oral. ${ }^{3}$

\section{Conclusão}

LPE é uma manifestação rara, porém subdiagnosticada e sub-relatada, de uma doença cutânea comum. Devido aos achados endoscópicos poderem ser sutis ou ausentes, a histologia pode ser inconclusiva, havendo muitas vezes um atraso significativo entre o início dos sintomas e o diagnóstico. ${ }^{27,31,38}$ A manutenção de um alto índice de suspeita no contexto clínico correto pode facilitar um rápido diagnóstico e o início precoce do tratamento, podendo alterar drasticamente o curso natural da doença.

Sugerimos que o LPE seja fortemente considerado no diagnóstico diferencial de mulheres de meia-idade que apresentam disfagia, 
odinofagia, ou ambos, ${ }^{1}$ com ou sem LP conhecido ou LP erosivo nos locais habituais das mucosas, ${ }^{2}$ com uma história ausente e/ou sintomas de refluxo gastroesofágico, manometria esofágica e estudos de $\mathrm{pH}$ normais, ${ }^{3}$ com resposta pobre ou mínima para os tratamentos habituais de esofagite de refluxo, e com endoscopia demonstrando lesões esofágicas proximais. ${ }^{4} \mathrm{Uma}$ prova terapêutica, resultando em melhora rápida com corticosteroides sistêmicos ou ciclosporina, também suporta o diagnóstico de LPE.

Tais pacientes devem ser encaminhados para um dermatologista para um exame completo com biópsias da mucosa e pele; e um gastroenterologista para endoscopia com biópsias esofágicas e avaliação de esofagite de refluxo. Uma abordagem multidisciplinar para a administração do caso é essencial.

Finalmente, sugerimos que os médicos encaminhem rapidamente os pacientes sintomáticos com LP para um gastroenterologista para realização de endoscopia digestiva alta e biópsias. Lembramos também a todos os médicos que a transformação maligna do LPE para CEC foi relatada e que o risco de degeneração maligna, embora desconhecida, pode ser ainda maior em pacientes com LP oral prévio associado a CEC. Recomenda-se vigilância endoscópica, em intervalos entre um a dois anos, especialmente em pacientes com LP oral grave ou LP oral associado a CEC.

\section{Referências}

1. Canto AM, Müller H, Freitas RR, da Silva Santos PS Líquen plano oral (LPO): diagnóstico clínico e complementar. An Bras Dermatol. 2010;85(5):669-75.

2. Lo Muzio L, Mignogna MD, Favia G, Procaccini M, Testa NF, Bucci E. The possible association between oral lichen planus and oral squamous cell carcinoma: a clinical evaluation on 14 cases and a review of the literature. Oral Oncol. 1998;34:239-46.

3. Abraham SC, Ravich WJ, Anhalt GJ, Yardley JH, Wu TT. Esophageal lichen planus: case report and review of the literature. Am J Surg Pathol. 2000;24:1678-82.

4. Rogers RS $3^{\text {rd }}$, Eisen D. Erosive oral lichen planus with genital lesions: the vulvovaginal-gingival syndrome and the penogingival syndrome. Dermatol Clin. 2003;21:91-8, vi-vii.

5. Harewood GC, Murray JA, Cameron AJ. Esophageal lichen planus: the Mayo Clinic experience. Dis
Esophagus. 1999;12:309-11.

6. Leyva-Leon F, Wright AL, Wight RG, Harrington Cl. Esophageal lichen planus presenting with dysphagia. Int J Dermatol. 1990;29:354-5.

7. Al-Shihabi BM, Jackson JM. Dysphagia due to pharyngeal and esophageal lichen planus. J Laryngol Otol. 1982;96:567-71.

8. Sheehan-Dare RA, Cotterill JA, Simmons AV. Esophageal lichen planus. Br J Dermatol. 1986;115:729-30.

9. Guedon C, Kuffer R, Thomine E, Lerebours E, Colin R. Stenosing lichen planus of the esophagus [in French]. Gastroenterol Clin Biol. 1982;6:1049-50.

10. Lefer LG. Lichen planus of the esophagus. Am J Dermatopathol. 1982;4:267-9.

11. Ottignon Y, Carayon P, Deschamps JP, Hirsch JP, Caille JP, Pageaut G. Stenosing lichen planus of the esophagus: gastroesophageal reflux does not appear to be the cause! [in French] Gastroenterol Clin Biol. 1983;7:830-1.

12. Lavignolle A, Renaut JJ, Le Bodic L. Stenosing erosive lichen planus of the esophagus [in French]. Gastroenterol Clin Biol. 1983;7:829-30.

13. Quevrin F, Simonis-Blumenfrucht A, Andre P, Saladhin A. Lichen plan plurimuqueux et atteinte oesophagienne. La Semaine des hôpitaux de Paris. 1986;62:1255-7.

14. Bousser A, Mosser C, Ramee M, Delambre C, Gosselin M, Chevrant-Breton J. Lichen plan et stenose oesophagienne. Ann Dermatol Venereol. 1986;113:938-9.

15. Plentz RR, Muller CC, Laenger F, Manns MP, Meier PN. Lichen planus esophagitis without skin, genital or oral mucosa involvement [in German]. Z Gastroenterol. 2004;42:379-82.

16. Schiavino D, Murzilli F, Forti G, Chiarelli C, Altomonte L, Magaro M. Mucocutaneous lichen planus with esophageal involvement: a clinical case [in Italian]. Minerva Med. 1992;83:73-6.

17. Manna R, Garcovich S, Giovinale M, Marinaro A, Manganelli C, Zampetti A, et al. Systemic complications of esophageal lichen planus. Int J Immunopathol Pharmacol. 2013 Apr-Jun;26(2):575-8.

18. Valdes F, Caparrini A, Calzada JM. Lichen planus with esophageal involvement [in Spanish]. Actas Dermo Sif. 2007;98:361-4.

19. Goñi ES, Arín LA, Vila CJJ, Jiménez PFJ, Ruiz-Clavijo GD, Carrascosa GJ, Almendral LML. Rituximab as rescue therapy in refractory esophageal lichen planus. Gastroenterol Hepatol. 2013 Apr;36(4):264-7

20. Oliveira JP, Uribe NC, Azulay-Abulafia L, Quintella LP. Líquen plano esofágico. An Bras Dermatol (in press).

21. Dickens CM, Heseltine D, Walton S, Bennett JR. The 
esophagus in lichen planus: an endoscopic study. BMJ 1990;300:84.

22. Menges M, Hohloch K, Pueschel W, Stallmach A. Lichen planus with esophageal involvement: a case report and review of the literature. Digestion. 2002;65:184-9.

23. Eisen D. The evaluation of cutaneous, genital, scalp, nail, esophageal, and ocular involvement in patients with oral lichen planus. Oral Surg Oral Med Oral Pathol Oral Radiol Endod. 1999;88:431-6.

24. Eisen D. The clinical features, malignant potential, and systemic associations of oral lichen planus: a study of 723 patients. J Am Acad Dermatol. 2002;46:207-14.

25. Calabrese C, Fabbri A, Benni M, Areni A, Scialpi C, Miglioli M, et al. Squamous cell carcinoma arising in esophageal lichen planus. Gastrointest Endosc. 2003;57:596-9.

26. Bigby M. The relationship between lichen planus and hepatitis C clarified. Arch Dermatol. 2009;145:1048-50.

27. Fox LP, Lightdale CJ, Grossman ME. Lichen planus of the esophagus: What dermatologists need to know. J Am Acad Dermatol. 2011;65:175-83

28. Ukleja A, DeVault KR, Stark ME, Achem SR. Lichen planus involving the esophagus. Dig Dis Sci. 2001;46:2292-7.

29. Chryssostalis A, Gaudric M, Terris B, Coriat R, Prat F, Chaussade S. Esophageal lichen planus: a series of eight cases including a patient with esophageal verrucous carcinoma; a case series. Endoscopy. 2008;40:764-8.

30. Quispel R, van Boxel OS, Schipper ME, Sigurdsson V, Canninga-van Dijk MR, Kerckhoffs A, et al. High prevalence of esophageal involvement in lichen planus: a study using magnification chromoendoscopy.
Endoscopy 2009;41:187-93.

31. Kirsch M. Esophageal lichen planus: a forgotten diagnosis. J Clin Gastroenterol. 1995;20:145-6.

32. Evans AV, Fletcher CL, Owen WJ, Hay RJ. Esophageal lichen planus. Clin Exp Dermatol. 2000;25:36-7.

33. Van der Meij EH, Mast H, van der Waal I. The possible premalignant character of oral lichen planus and oral lichenoid lesions: a prospective five-year follow-up study of 192 patients. Oral Oncol. 2007;43:742-8.

34. Silverman S Jr, Gorsky M, Lozada-Nur F, Giannotti K. A prospective study of findings and management in 214 patients with oral lichen planus. Oral Surg Oral Med Oral Pathol. 1991;72:665-70.

35. Markopoulos AK, Antoniades D, Papanayotou P, Trigonidis G. Malignant potential of oral lichen planus; a follow-up study of 326 patients. Oral Oncol. 1997;33:263-9.

36. Xue JL, Fan MW, Wang SZ, Chen XM, Li Y, Wang L. A clinical study of 674 patients with oral lichen planus in China. J Oral Pathol Med. 2005;34:467-72.

37. Cohan DM, Popat S, Kaplan SE, Rigual N, Loree T, Hicks WL Jr. Oropharyngeal cancer: current understanding and management. Curr Opin Otolaryngol Head Neck Surg. 2009;17:88-94.

38. Scully C, Bagan J. Oral squamous cell carcinoma: overview of current understanding of etiopathogenesis and clinical implications. Oral Dis. 2009;15:388-99.

39. Hietanen J, Paasonen MR, Kuhlefelt M, Malmstrom $M$. A retrospective study of oral lichen planus patients with concurrent or subsequent development of malignancy. Oral Oncol. 1999;35:278-82.

40. O'Neill ID. Off-label use of biologicals in the management of inflammatory oral mucosal disease. I Oral Pathol Med. 2008;37:575-81.

\section{Janine Pichler de Oliveira}

Instituto de Dermatologia Professor Rubem David Azulay. Santa Casa da Misericórdia. Rio de Janeiro, RJ, Brasil.

\section{Luna Azulay-Abulafia}

Departamento de Especialidades Médicas. Serviço de Dermatologia. Hospital Universitário Pedro Ernesto. Faculdade de Ciências Médicas. Universidade do Estado do Rio de Janeiro. Rio de Janeiro, RJ, Brasil. 